the adverse events were almost tolerable. However, we should interpret the result of clinical and endoscopic response with caution.

\section{IDDF2020-ABS-0023 ABDOMINAL TUBERCULOSIS: SURGICAL MANAGEMENT OF PERFORATED INTESTINAL ULCERS IN PATIENTS WITH HIV/TB}

${ }^{1}$ Mikhail Reshetnikov*, 'Dmitriy Plotkin, ${ }^{1}$ Mikhail Sinitsyn, ${ }^{2}$ Evgeniy Stepanov. ${ }^{1}$ Moscow Research and Clinical Center for TB Control, Russia; ${ }^{2}$ Pirogov Russian National Research Medical University, Russia

\subsection{6/gutjnl-2020-IDDF.46}

Background The growth tendencies of abdominal tuberculosis have increased more than twice between the year 2006-2016, and primarily associated, with an increase in the number of HIV-positive individuals. The most formidable and most frequent complication of tuberculosis of the abdominal cavity is perforation of specific ulcers of the intestine.

Methods Was to evaluate the results of surgical management of patients with perforated tuberculosis ulcers of the intestine against the background of late-stage of HIV infection and to develop optimal surgical tactics for this category of patients. 149 patients with perforated tuberculosis ulcers of the intestine underwent surgical treatment at the surgical department of our clinic in the period of time between the year 2006 and 2016.

Results Ulcerative lesions of intestine were detected in all cases during laparotomy, single and multiple ulcers were located in the jejunum, iliac or cecum, more often affecting the ileocecal zone. The most optimal method of surgical management of perforated ulcers of the intestine in patients with the late stages of HIV infection is performing a resection of the affected portion of the intestine with the construction of delayed anastomosis.

Conclusions A resurgence in tuberculosis during the HIV era produces a new spectrum of presentations for the surgeon. Avoidance of construction of primary anastomosis in conditions of torpid peritonitis, as well as direct visual control during laparotomy, allows timely detection and elimination of new perforations, as well as evaluation of treatment effectiveness.

(Figure 1)

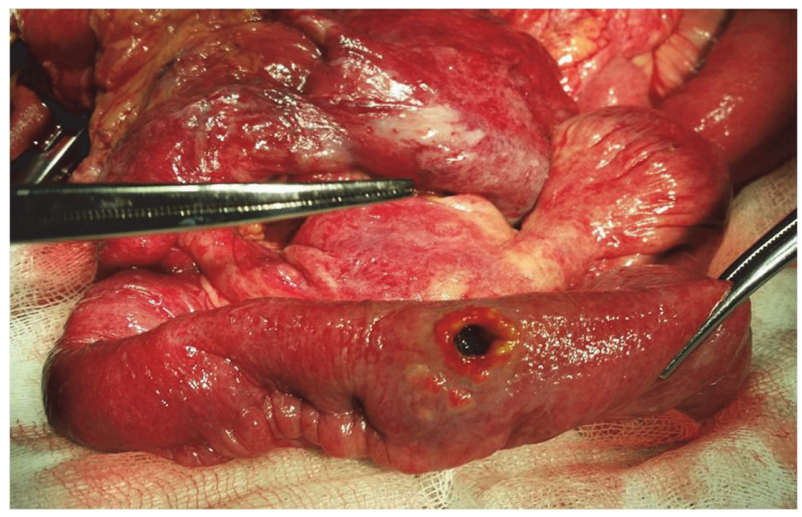

Abstract IDDF2020-ABS-0023 Figure 1 Perforated tubercular ulcer of ileum

\section{IDDF2020-ABS-0026 ULTRA-MUTATED PATIENTS WITH POLE OR POLD1 MUTATIONS EXHIBITS DISTINCT PATTERN BETWEEN RACES AND PRIMARY SITES IN COLORECTAL CANCER (CRC)}

${ }^{1}$ Wen Cai ${ }^{*},{ }^{1}$ Dehao $\mathrm{Wu},{ }^{2} \mathrm{Li}$ dong Wang, ${ }^{1}$ Shu Zheng, ${ }^{3}$ Hanguang $\mathrm{Hu}$, 'Weiting Ge. ${ }^{1}$ Cancer Institute (Key Laboratory of Cancer Prevention and Intervention, China National Ministry of Education), the Second Affiliated Hospital, School of Medicine, Zhejiang University, China; ${ }^{2}$ Henan Key Laboratory for Esophageal Cancer Research of the First Affiliated Hospital, State Key Laboratory for Esophageal Cancer Prevention and Treatment, Zhengzhou University, China; ${ }^{3}$ Department of Medical Oncology, the Second Affiliated Hospital, School of Medicine, Zhejiang University, China

\subsection{6/gutjnl-2020-IDDF.47}

Background POLE/POLD1 mutation leads to ultra-mutated phenotype in colorectal cancer (CRC) and could be a promising marker for immunotherapy.

Methods We sequenced 338 CRC patients in Asian and obtained 284 white CRC patients from TCGA.

Results The prevalence of POLE/POLD1 mutations were different in racial $(6.51 \%$ vs $11.27 \% ; P=0.036)$ (table 1$)$. The right-sided colon shows the highest rate of POLE/POLD1 mutations in both group $(50 \%$ vs $65.63 \%, P=0.251)$, while Asian has a higher rate in the left-sided colon than White (36.40\% vs $9.38 \%, P=0.016)$. We further calculate the prevalence of POLE/POLD1 mutation in different primary sites

Abstract IDDF2020-ABS-0026 Table 1 The differences of POLE/ POLD1 mutation in CRC

\begin{tabular}{|c|c|c|c|}
\hline & Asian $(n=22 / 338)^{*}$ & White $(n=32 / 284)^{*}$ & $P$-value \\
\hline Age (Mean) & $56.36(49.82-62.90)$ & $65.44(60.65-70.23)$ & 0.422 \\
\hline Male, n (\%) & $11(50.00)$ & $17(53.13)$ & 0.821 \\
\hline Family history, $\mathrm{n}(\%)$ & $5(22.73)$ & $5(26.32)$ & 1.000 \\
\hline MSI-H, n (\%) & $11(50.00)$ & $21(45.70)$ & 0.737 \\
\hline mutPerMB(Mean) & $118.82(77.58-160.05)$ & $62.88(39.30-86.46)$ & 0.436 \\
\hline TNM stage, n (\%) & & & 0.387 \\
\hline I & $1(4.55)$ & $5(15.63)$ & \\
\hline II & 15(68.18) & $20(62.50)$ & \\
\hline III & $6(27.27)$ & $5(15.63)$ & \\
\hline IV & $0(0.00)$ & $1(3.13)$ & \\
\hline \multicolumn{4}{|l|}{ Primary site } \\
\hline Right-sided, n (\%) & $11(50.00)$ & $21(65.63)$ & 0.251 \\
\hline Cecum & & 9 & \\
\hline Ascending Colon & 6 & 7 & \\
\hline Hepatic Flexure & 1 & 1 & \\
\hline Transverse Colon & 2 & 4 & \\
\hline Splenic Flexure & 2 & & \\
\hline Left-sided, n (\%) & $8(36.40)$ & $3(9.38)$ & 0.016 \\
\hline Descending Colon & 4 & & \\
\hline Sigmoid Colon & 4 & 3 & \\
\hline Rectum, n (\%) & $3(13.6)$ & $5(15.63)$ & 0.851 \\
\hline Rectosigmoid Junction & & 1 & \\
\hline Rectum & 3 & 4 & \\
\hline $\begin{array}{l}\text { With muts, n/All Right-sided, } \\
\text { n }(\%)^{* *}\end{array}$ & 11/94(11.70) & $16 / 109(14.68)$ & 0.533 \\
\hline $\begin{array}{l}\text { With muts, n/All Left-sided, } \\
\mathrm{n}(\%)^{* *}\end{array}$ & $9 / 87(10.35)$ & $1 / 66(1.51)$ & 0.027 \\
\hline $\begin{array}{l}\text { With muts, n/All Rectum, } n \\
(\%)^{* *}\end{array}$ & $3 / 157(1.90)$ & $5 / 68(7.40)$ & 0.102 \\
\hline
\end{tabular}

